\title{
El rol de los hombres en enfermería: una revisión histórica-narrativa
}

\section{The Role of Men in Nursing: A Historical, Narrative Review}

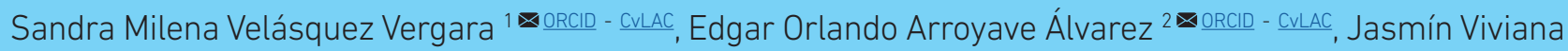
Cacante Caballero 3 ORCID - $\underline{\text { CVLAC }}$

Fecha correspondencia:

Recibido: 6 de marzo de 2021.

Aceptado: 9 de abril de 2021.

Forma de citar:

Velásquez-Vergara S, Arroyave-Álvarez E, Cacante-Caballero J. El rol de los hombres en enfermería: una revisión histórica-narrativa. Revista CES Enfermería, 2021; 2(1): $21-34$.

Open access

Creative commons

Ethics of publications

Peer review

Open Journal System

DOl: http://dx.doi.org/10.21615/

cesenferm.2.1.2

ISSNe 2745-049X

Filiación:

1. Enfermera, Especialista en Gerencia de la Calidad y Auditoría en Salud, PhD(c) en Enfermería, Universidad de Antioquia. Miembro del grupo de investigación Psicología, Sociedad y Subjetividades (U. de A.) Medellín, Antioquia.

2. Psicólogo, Magíster en Filosofía, PhD en Ciencias Sociales, Docente asociado de la Facultad de Ciencias Sociales y Humanas. Universidad de Antioquia. Miembro del grupo de

\section{Resumen}

Objetivo: Visibilizar el papel de los hombres en el desarrollo de la enfermería desde una perspectiva histórica-narrativa. Metodología: Revisión integrativa de la literatura en ocho bases de datos con la siguiente pregunta: ¿cuál ha sido el rol de los hombres en enfermería y sus aportes al desarrollo disciplinar? Los elementos seleccionados fueron analizados por los autores de manera detallada para dar respuesta a la inquietud planteada. Resultados: Después de aplicar los criterios de inclusión y exclusión, se seleccionaron y analizaron veinticinco artículos que posibilitaron explorar, describir y discutir de manera amplia sobre el rol de los hombres en enfermería a partir de una mirada histórica-narrativa. Conclusiones: La revisión permite reflexionar sobre el rol que han desempeñado los hombres dentro de la enfermería, a través de la historia y la importancia de su presencia a nivel disciplinar en términos de igualdad de género, tomando como referentes los planteamientos de exponentes de las ciencias sociales y de la enfermería.

Palabras clave: rol; enfermeros; hombres.

\section{Abstract}

Objective: To make, the role of men in the development of nursing from a historical-narrative perspective, visible. Methodology: Narrative review of the literature in eight databases with the following question: What has been the role of men in nursing and their contributions to disciplinary development? The selected elements were analyzed by the authors in detail to respond to the concern raised. Results: After applying the inclusion and exclusion criteria, 25 articles were selected and analyzed, which made it possible to explore, describe and discuss broadly the role of men in nursing from a historical perspective. Conclusions: The review allows us to reflect on the role that men have played in nursing throughout history and the importance of their presence at the disciplinary level in terms of gender equality, taking as references the approaches of exponents of the Social Sciences and Nursing.

Keywords: role; nurses; men. 
investigación Psicología, Sociedad y Subjetividades (U. de A.). Medellín Antioquia.

3. Enfermera. Especialista en Cuidado al Niño en Estado Crítico, Magíster en Enfermería, PhD en Bioética, docente de la facultad de enfermería, Universidad de Antioquia. Colombia.

\section{Introducción}

Tradicionalmente, el rol es comprendido como el papel o función que un individuo cumple en determinada sociedad, basado en un sistema de valores que guían y dirigen la conducta de cada individuo. En ese orden de ideas, en virtud de lo que se considera adecuado o no, tanto para hombres como para mujeres, emergen estereotipos para nombrar aquello considerado habitual para ambos sexos.

En su conceptualización, el rol ha sido abordado desde distintas disciplinas. No obstante, para fines de este artículo, se acogerá la definición propuesta por Mendes y De Almeida (1), quienes han analizado la teoría de roles en el contexto de enfermería. Según estas investigadoras, el rol es un grupo de patrones, descripciones o normas aplicadas a los comportamientos de una persona en virtud de la posición que ocupa dentro de determinada sociedad.

Ahora bien, si analizamos el rol desde una perspectiva de género, es frecuente hallar publicaciones que mencionan las dificultades que enfrentan las mujeres al ingresar a profesiones o empleos predominantemente masculinos, situación que ocurre escasamente con los hombres. En este sentido, encontrar información acerca de los roles profesionales de los enfermeros no es tarea fácil, debido a que los estudios relacionados a las experiencias de este grupo poblacional son pocos $(2,3,4)$. Pese a ello, la historia ha mostrado que cuidar es una acción intrínseca a la experiencia humana y que también ha sido ejercida ampliamente por los hombres a lo largo de los siglos en diferentes contextos (religioso, militar, clínico, comunitario).

Esta es la razón por la cual esta revisión histórica cobra importancia, al reconocer el papel de los hombres en el desarrollo disciplinar y profesional de la enfermería. Así pues, la intención con este trabajo es promover en las futuras generaciones de hombres enfermeros, un empoderamiento que conlleve a una participación activa y visible en diferentes escenarios disciplinares y profesionales.

Otro de los aportes importantes de este trabajo y de otros similares es abrir la posibilidad de que se contemple, dentro de los contenidos curriculares en enfermería, una formación histórica que dé a conocer las contribuciones hechas por los hombres, no solo en materia de cuidado, sino también para la evolución de la enfermería. Lo anterior daría como resultado, en el momento actual y hacia el futuro, una visión "neutral" de la enfermería, que promueva la vinculación de hombres y mujeres por igual, demostrando que el cuidado no es un asunto generizado, sino una actividad de hombres y de mujeres que tiene como objetivo el cuidado de la vida.

\section{Material y método}

Con el ánimo de abordar de la manera más completa la temática de estudio, se llevó a cabo una revisión integrativa de la literatura, teniendo como referente cronológico, material académico producido en los últimos diez años (2010-2020). A nivel disciplinar, este método se considera valioso, dado que permite revisar el conocimiento disponible de un asunto determinado y los vacíos existentes del mismo (5). Los descriptores que guiaron la búsqueda fueron: rol, enfermeros, hombres. La estrategia de investigación se basó en la siguiente combinación: "Role" OR "nursing Men" OR "Nurse" AND "Men Nursing" OR "Role". Inicialmente, se realizó una búsqueda abierta en Google Scholar ${ }^{\circledR}$, obteniéndose 22000 elementos. Posteriormente, se consultaron las bases de datos MedLine, SAGE, PubMed, SCOPUS, Taylor \& Francis, BVS y ClinicalKey. En total, se obtuvieron 26571 referencias y de esta selección, se revisaron 104 artículos 
en forma integral (Figura 1). Se excluyó el material: en idiomas distintos al inglés, el español y el portugués; artículos de acceso restringido; artículos duplicados; y textos que no mencionaban los descriptores propuestos.

El análisis de los estudios seleccionados estuvo a cargo de los autores. Se elaboraron cuadros de referencias, con la intención de organizar un único archivo de texto. De esta forma, en total, se analizaron 25 artículos. En la Tabla 1, se presenta una síntesis de estos.

Tabla 1. Síntesis de estudios encontrados.

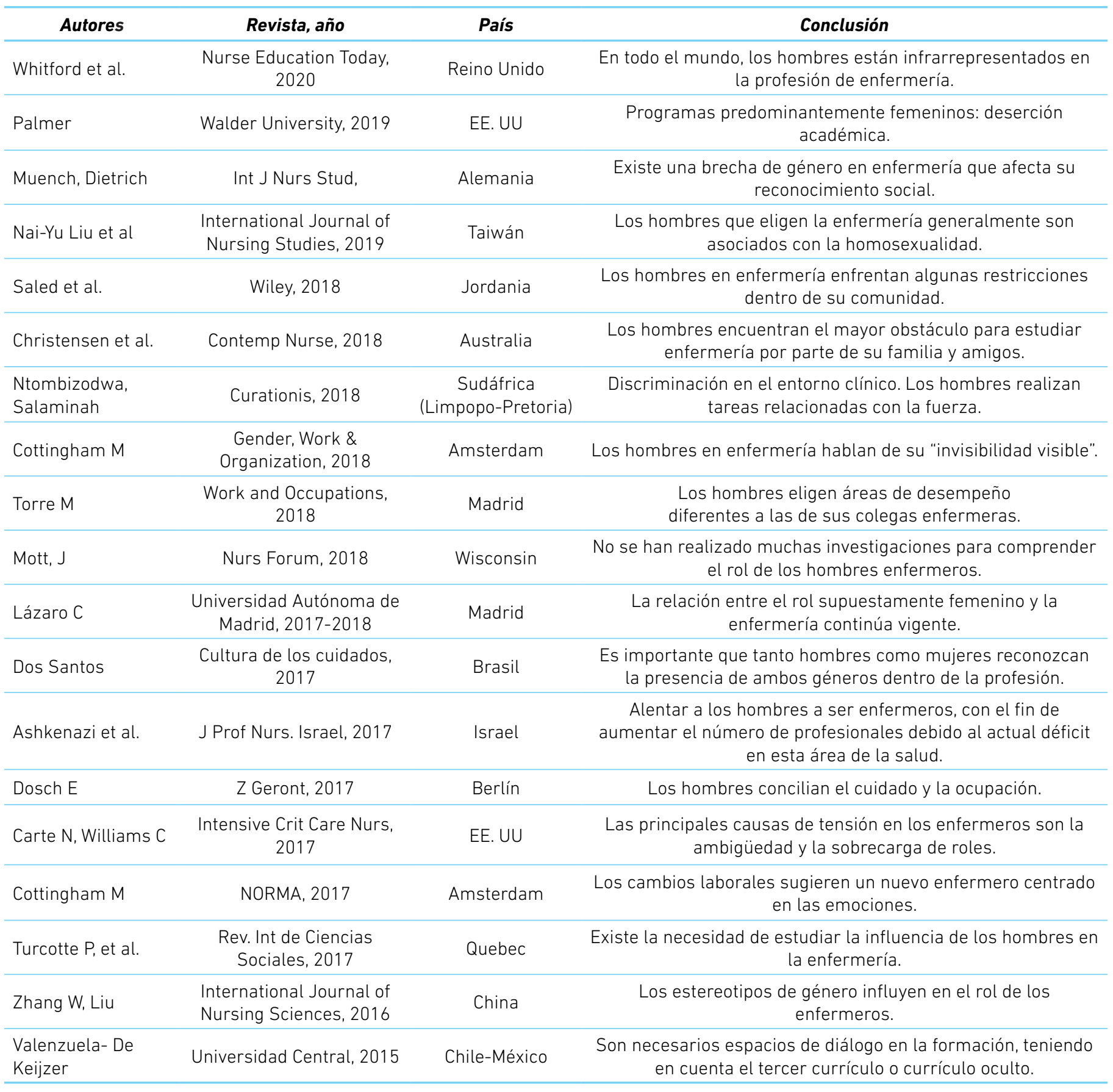


Enero - junio 2021 - Página 24

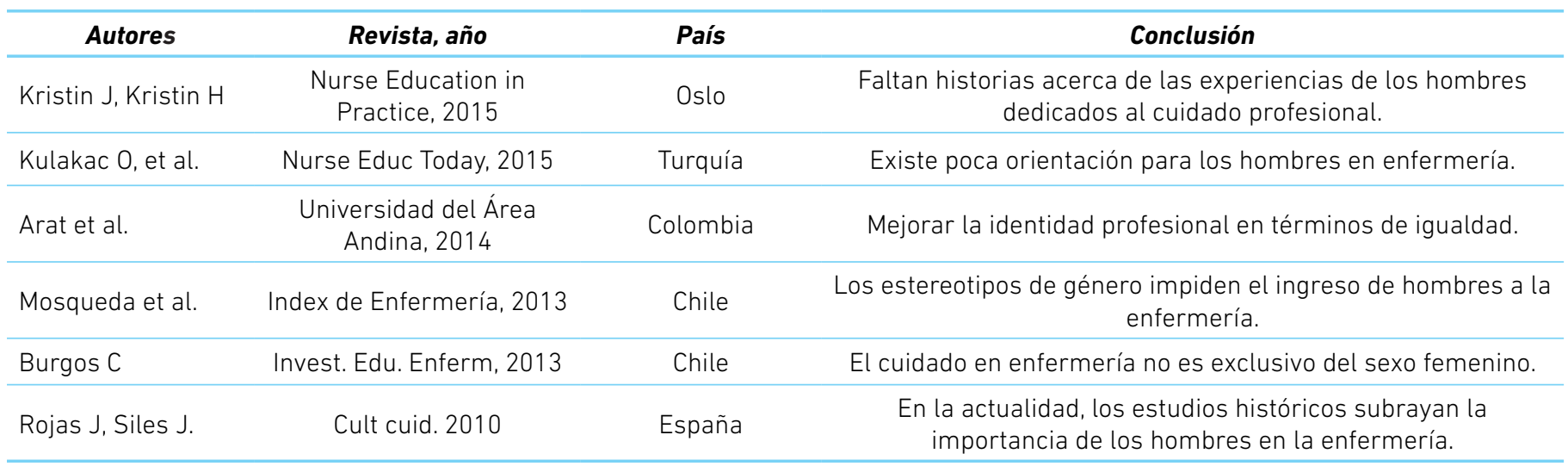

Fuente: elaboración propia.

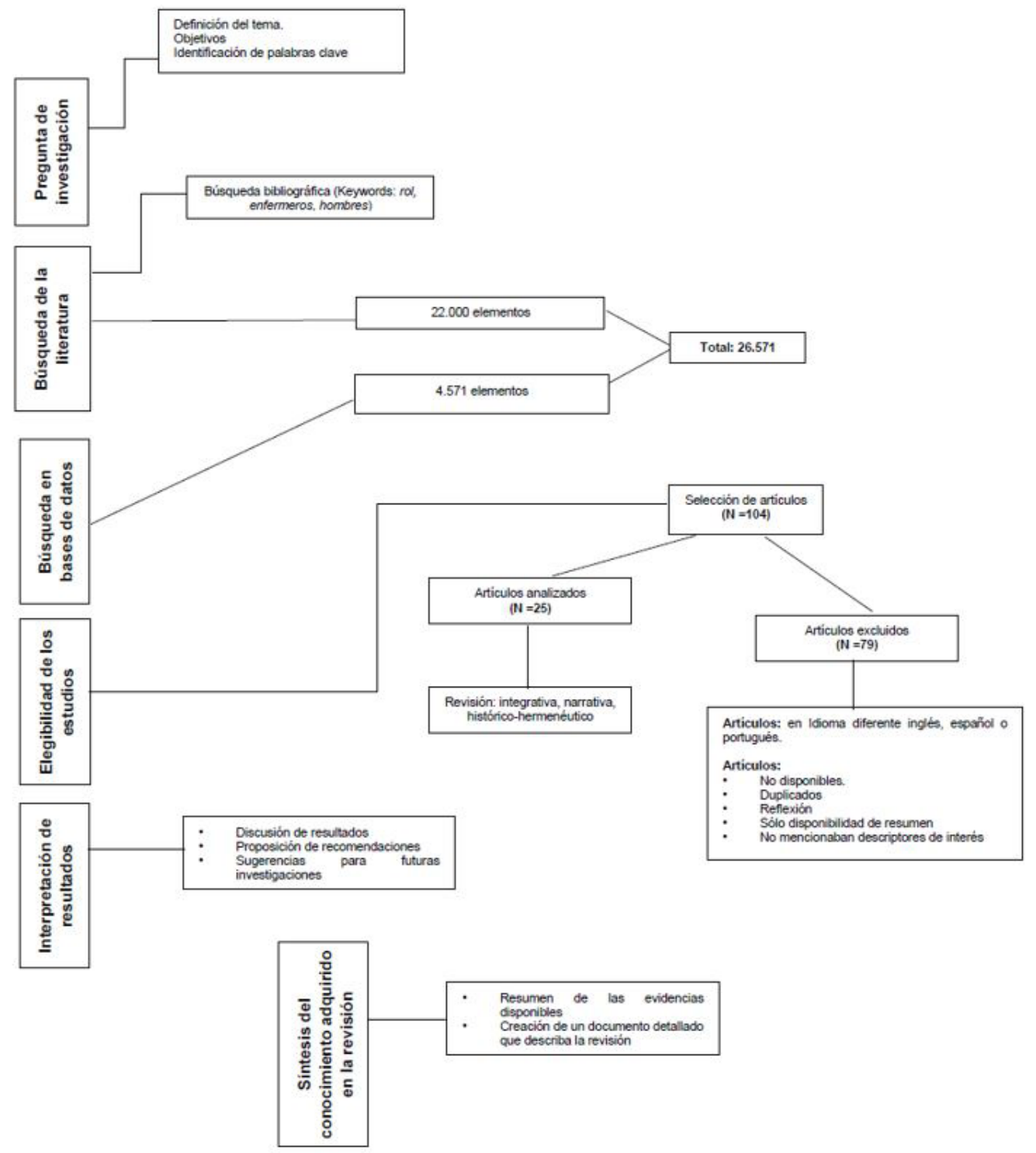

Figura 1. Esquema de búsqueda bibliográfica. Adaptado de: Dal Sasso, Karina, De Campos Renata, Galvão, Cristina. Revisão integrativa: método de pesquisa para a incorporação de evidências na saúde e na enfermagem. Texto \& Contexto - Enfermagem. 2008;17(4): 758-64. 


\section{Resultados}

Para dar respuesta al objetivo planteado de conocer el rol de los hombres en la profesión de enfermería desde una perspectiva histórico-narrativa, iniciamos este recuento con lo expuesto por Marie-Françoise Collière (6), quien afirma que las prácticas de cuidado, desde sus inicios, tuvieron diferenciación con relación al género. Esto se evidencia en el hecho de que la prácticas alrededor de la vida y las desarrolladas al interior del hogar son propias de las mujeres, mientras que las curas del cuerpo físico, su exploración y la introducción de tecnología fueron y siguen siendo consideradas como actividades de hombres, así como aquellos cuidados que exigen de la fuerza física, como reducción de fracturas y el dominio físico de personas que presentan síntomas de trastornos mentales (Figura 2).

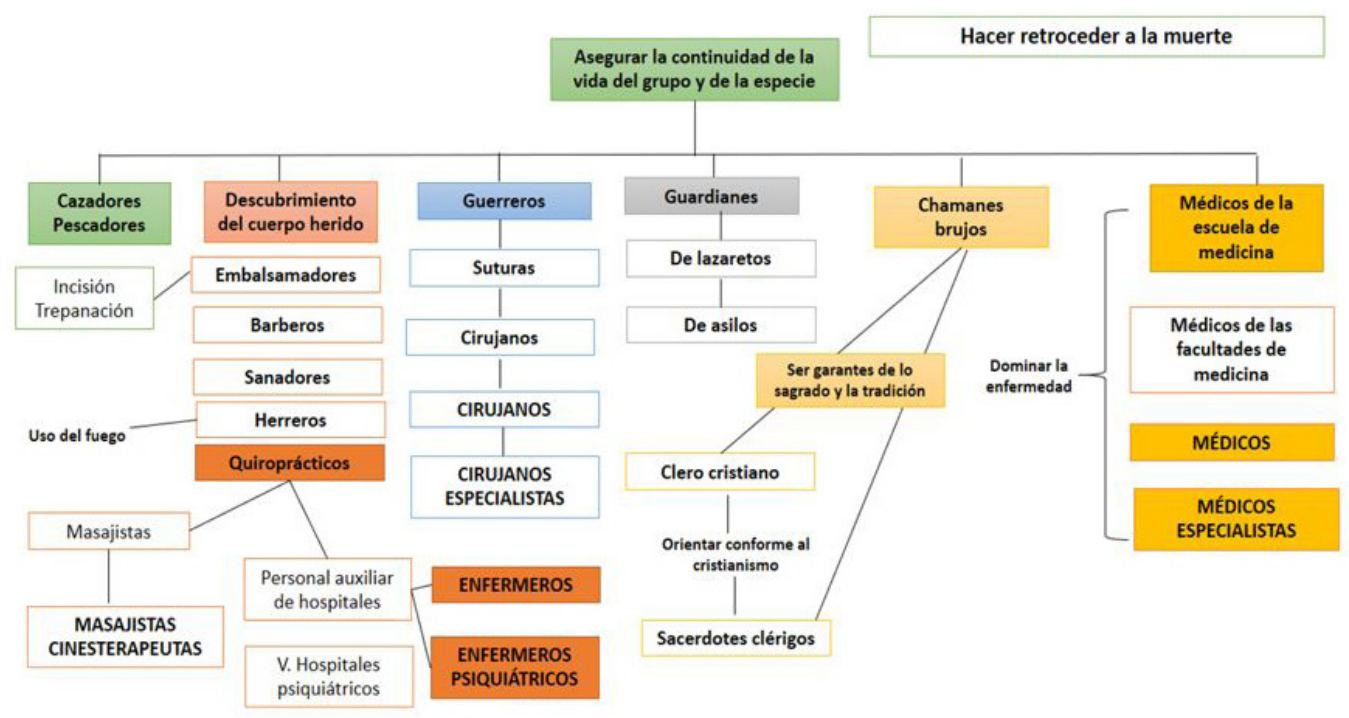

Figura 2. Prácticas cuidadoras aseguradas por los hombres (6). Adaptado de: Collière M. Origen de las prácticas de cuidadoras; su influencia en la práctica de la Enfermería. En: Promover la vida. Segunda Edición. México: McGraw-Hill Interamericana; 2009. p. 5-13.

En consideración a lo anterior, es importante destacar que la división de los cuidados ha dado lugar a diversas formas de ejercicio como enfermeros vinculados al ejército y órdenes religiosas influenciadas por el cristianismo, las cuales dejaron un legado de solidaridad y respeto, interpretando el cuidado como una virtud y un medio para alcanzar la salvación (7). Entre las órdenes religiosas que han sobresalido históricamente, podemos nombrar: (a) los Caballeros Hospitalarios, institución que se caracterizó por combatir el islam y por fundar hospitales; (b) los Caballeros de San Lázaro, dedicados a atender a personas que padecían lepra (8); y (c) los Caballeros Teutónicos, quienes realizaban votos de castidad, obediencia, pobreza y un voto especial que los obligaba a cuidar los enfermos $(9,10)$.

En el seno de estas comunidades, se destacaron varios hombres, tal es el caso de Camilo Lelis, reconocido por trabajar como enfermero y por ser el creador de la orden de "Los Ministros de los Enfermos", conocida como "los Camilos" (11). Asimismo, en el contexto latinoamericano, a mediados del siglo XVI, el fraile mexicano Juan de Mena trabajó arduamente por el cuidado de los enfermos (12). En Colombia, ha sido valorada la obra del sacerdote y jesuita español Pedro Claver, reconocido por algunos investigadores como el "padre de la enfermería en Colombia" $(13,14)$. 
Otros han alcanzado su liberación, como el esclavo negro James Derham, en Nueva Orleans en 1783, quien dedicándose al cuidado de los demás, motivado por la compasión más que por la salvación del alma, logró ser ciudadano libre; más tarde, alcanzó el título de médico siendo el primer galeno de color de los Estados Unidos (15).

Otro hombre que se destacó en materia de cuidado fue el filántropo y pastor alemán Theodor Fliedner: a principios del siglo XIX, fundó el instituto de Kaiserswerth, establecimiento reconocido a nivel mundial como el primer sitio destinado a la formación de mujeres dedicadas al cuidado, denominadas Diaconisas, quienes consagraban su vida al servicio de los demás. Entre sus múltiples visitas, debido a su distinción mundial, estuvo Florence Nightingale, quien siguiendo el modelo de Fliedner, desarrolló en Londres un programa de enfermería similar (16). Asimismo, el activista humanitario Henry Dunant, inspirado en la obra de Nightingale, creó la Cruz Roja, organismo internacional de carácter neutral para la atención y cuidado de combatientes heridos en guerra (17).

Otro ejemplo del papel de los hombres en materia de cuidados es Walt Whitman, poeta y escritor del XIX; se desempeñó como enfermero voluntario en Washington durante la guerra civil. Whitman plasmó sus experiencias en una colección de poemas llamada Drumtaps y en su diario Specimen days and collect, pero su trabajo como enfermero lo inmortalizó en el poema The wound dresser (18).

Como hemos visto, en este corto recorrido, los hombres han contribuido al cuidado y su profesionalización en diferentes contextos, a pesar del poco reconocimiento de su labor. Precisamente, en búsqueda de ese reconocimiento en la enfermería - puesto que se trata de una profesión feminizada, lo que conlleva estereotipos negativos hacia los enfermeros-, en 1971, bajo la dirección de Steve Miller, surgió la AAMN (por sus siglas en inglés, American Association for Men In Nursing), cuyo propósito era proporcionar a los hombres un lugar de encuentro y discusión sobre factores que afectaban su desempeño debido a prácticas de discriminación y exclusión social (19) (Figuras 3 y 4).

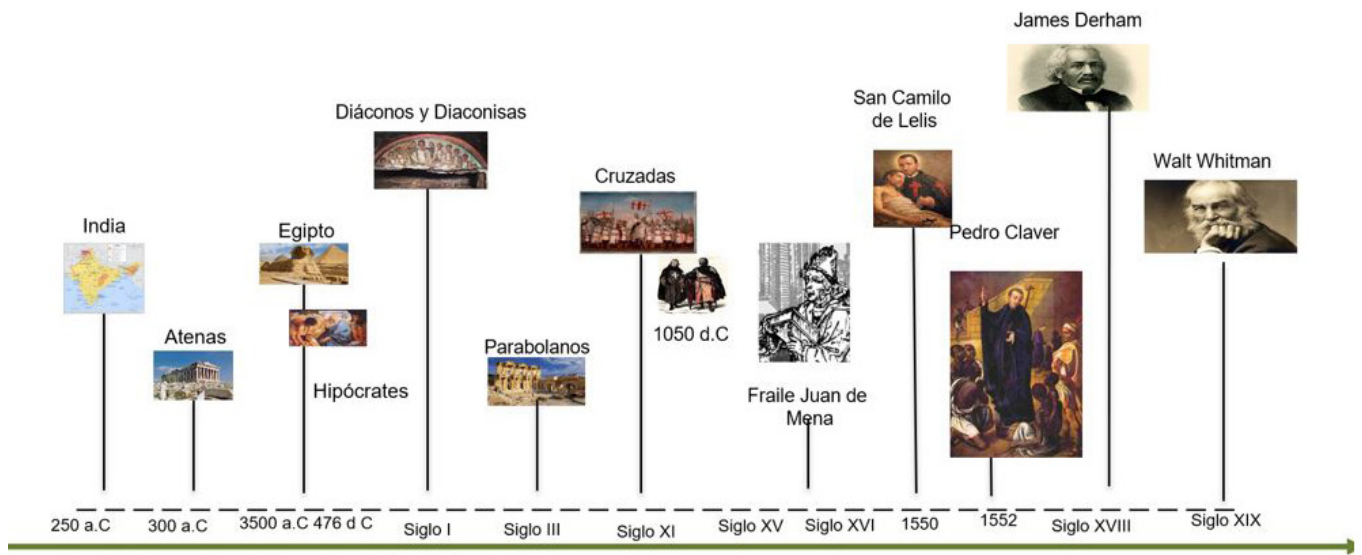

Figura 3. Presencia masculina en la historia de los cuidados (7-18). Fuente: elaboración propia. 


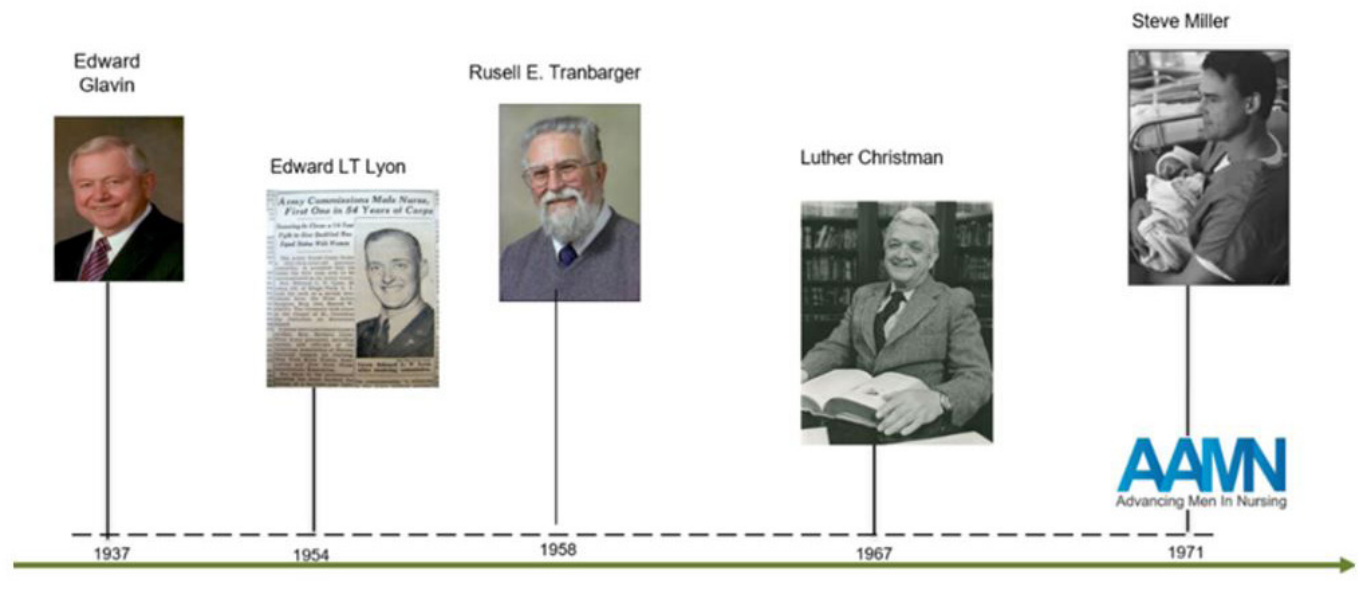

Figura 4. Presencia masculina en la historia de los cuidados (19). Fuente: elaboración propia.

Autores como Ashkenazi et al. (20) afirman que los varones eligen esta profesión por razones similares a sus colegas femeninas, pero una vez inician su formación, perciben que los espacios educativos están "diseñados para mujeres", lo que conlleva a moderar ciertos comportamientos tradicionalmente considerados masculinos para adaptarse al contexto. En esta misma línea, estudios como el de Turcotte et al. (21) señalan que los hombres tienden a especializarse en áreas con mayor "prestigio" y "autonomía", y evitan desempeñarse en áreas que guarden mayor relación con la "feminidad". En esta misma línea, Cottingham (22) asegura que los enfermeros prefieren áreas de bajo contacto físico y alta tecnología como un mecanismo para enmascarar los sentimientos de dolor, compasión y entrega, que podrían ser interpretados socialmente como muestra de debilidad, considerados tradicionalmente propios del género femenino. Las áreas donde generalmente se ubican los hombres son emergencias, terapia física, cuidados intensivos, terapia ocupacional y especialidades como enfermería cardiovascular y anestésica (23), áreas de desempeño que los aleja de la clásica visión de vocación y sensibilidad que históricamente ha distinguido a la profesión.

En relación con lo anterior, en su disertación doctoral, Palmer (24) concluye que, dentro de las facultades de enfermería, falta neutralidad frente al trato que reciben los hombres y una orientación laboral de acuerdo con sus expectativas profesionales. Asimismo, quienes logran ingresar a la formación académica, a menudo se ven expuestos a nivel familiar a expresiones estereotipadas como "ángel con un vestido blanco", "hermana", "matrona", que no solo resultan incómodas para los varones, sino que también los lleva a experimentar vergüenza (25).

Las situaciones descritas, además de generar deserción académica, también conllevan a los enfermeros a negociar percepciones contradictorias de los roles de género y enfrentarse con la disparidad de desempeñar un rol considerado exclusivamente femenino que poco coincide con los modelos hegemónicos de un hombre real (26). Esta condición genera pérdida de identidad profesional e inseguridad en los individuos, pues representan un papel que supuestamente no les corresponde (27). 
En síntesis, las normas y reglas sociales y de género, a la hora de brindar cuidado, restringen a los hombres en su ejercicio profesional e impiden el contacto con el otro, puesto que, en muchas oportunidades, tales manifestaciones son interpretadas de manera errónea en la mayoría de las ocasiones, al ser vinculadas con la orientación sexual del profesional (28).

\section{Discusión}

De acuerdo con el recorrido histórico planteado en los resultados, algunos aportes significativos de los hombres en el desarrollo disciplinar y a la formación académica, desde sus inicios hasta la fecha, guardan relación con la educación en diferentes contextos; por ejemplo, a nivel vocacional en el contexto colombiano, las primeras enseñanzas sobre higiene y cuidado de los enfermos fueron impartidas por religiosos pertenecientes a las comunidades de los Dominicos, Mercedarios, Capuchinos, Hermanos Hospitalarios de San Juan de Dios y Jesuitas, que, según Castrillón (29), estos monjes vinieron al país con el propósito de catequizar indígenas.

Los valores vocacionales de estas órdenes religiosas, como la obediencia, la confidencialidad, la entrega, la compasión y la continuidad en el cuidado, continúan vigentes en la enfermería y se reflejan en el Código Deontológico de Enfermería, en la Ley 266 y en la Política Nacional de Talento Humano en Enfermería y Plan Estratégico 2020-2030 (30-32).

Por otra parte, en un entorno laico, pero apoyados en valores similares a los mencionados antes, Theodor Fliedner y su esposa, como ya se dijo, fundaron la escuela de Diaconisas de Kaiserswerth, con la intención de formalizar el aprendizaje del cuidado. Esta institución contó con alumnas destacadas como Florence Nightingale, quien empleó una metodología similar a la de estos esposos en la formación de sus alumnas.

Asimismo, entre los aportes más importantes de los hombres en enfermería, se encuentra la formulación de teorías. Por ejemplo, Phil Barker, interesado por el agua como principal elemento de la vida y aprovechando su experiencia laboral como "auxiliar en un manicomio", hizo una analogía de la mente como un "océano", donde sus aguas se mueven y fluctúan. Este hecho dio como resultado la creación del modelo de la marea en la recuperación de la salud mental, donde Barker se enfocó en el cuidado de enfermería a pacientes psiquiátricos (33).

Otro hombre que también ha aportado al desarrollo disciplinar es James H. Husted, quien, de manera conjunta con su esposa, crearon la teoría bioética sinfonológica. Husted, con formación militar y con un fuerte interés por la obra de Spinoza, definió la sinfonología como el estudio de acuerdos bioéticos y los elementos necesarios para constituirlos. Este modelo vigente es utilizado como guía en el comportamiento del profesional de enfermería durante el desempeño de su rol (34).

Además de estos autores, hombres como Chad O' Lynn y Russell E. Tranbarger se han comprometido por visibilizar la presencia masculina dentro de la enfermería, construyendo textos académicos que dan a conocer los cambios y las oportunidades para los hombres a lo largo de la historia a nivel disciplinar (35). 
Lo anterior evidencia que los hombres han hecho grandes contribuciones al desarrollo y evolución de la enfermería. Sin embargo, poco se reconocen socialmente estos hechos. Razón por la cual, representantes como Luther Christman y Steve Miller, se han esmerado por trabajar y fortalecer asociaciones gremiales como la AAMN, con el fin de velar por los derechos y oportunidades para los hombres dentro de la profesión (36).

La revisión de literatura realizada en esta investigación permitió establecer que existen vacíos de estudios y reflexiones sobre el tema abordado $(37,38)$, pero, contrariamente, es frecuente hallar producción científica relacionada a la percepción cultural de la orientación sexual de los enfermeros $(39,40)$, lo que conlleva a un escaso ingreso de los hombres a la profesión y una inclinación mayor a áreas que perciben altamente "tecnológicas", como una manera de combatir el estigma social.

Podría afirmarse, entonces, a juzgar por la división sexual del trabajo, que las sociedades conciben ciertos roles como masculinos y femeninos. En el caso de la enfermería, los hombres ocultan expresiones de ternura y compasión, que van ligadas al cuidado; en consecuencia, en algunas ocasiones, adoptan actitudes estereotipadas del hombre rudo y beligerante de acuerdo con los patrones heteronormativos vigentes (41).

\section{Conclusiones}

Este trabajo hace un acercamiento histórico al rol de los hombres en el cuidado y dentro de la enfermería, desde una perspectiva de género, visibilizando los aportes de algunos hombres al cuidado tanto en forma empírica como profesional. Asimismo, esta revisión documental destaca que, a pesar del desconocimiento de la importancia de los hombres a la enfermería, estos han contribuido en el campo docente, investigativo y teórico, aportando al desarrollo y consolidación de esta profesión.

También, se puede afirmar que existen vacíos sobre este tema a nivel mundial, regional y local que visibilicen los aportes que distintos hombres han brindado al desarrollo de la profesión, especialmente en Latinoamérica y el Caribe, donde aún no se han encontrado exponentes masculinos, como, contrariamente, ha sucedido con destacadas mujeres en la enfermería en todo el mundo.

No obstante, este rastreo posibilitó identificar el trabajo de algunos hombres, en particular en Colombia, quienes continúan aportando a la disciplina, como John Henry Osorio, Camilo Duque, o Víctor Quintero (42-44), destacados no solo por su producción científica, sino por su contribución a distintas fases de la formación profesional. Valorar los aportes de estos hombres, en materia de generación de conocimiento, no solo permite enriquecer los currículos de enfermería desde otra postura, sino que favorece el desarrollo y avance de la profesión (8).

Una evidencia del cambio de los roles es el desempeño de los hombres en cargos directivos o de asociaciones de enfermería como los docentes Juan Guillermo Rojas, quien ejerce un cargo administrativo en la Organización Colegial de Enfermería (45), o León Denis Velosa, el primer hombre en dirigir una Facultad de Enfermería en Antioquia, Colombia (46).

Reconocer la labor de estos y muchos otros hombres resulta relevante, dado que su trabajo inspira a las futuras generaciones de enfermeros a continuar avanzando en su formación, pues tendrán referentes dentro de la profesión. 
Finalmente, se quiere añadir que el cambio de paradigmas y la ruptura de estereotipos de género en la enfermería no solo beneficia a la profesión en términos de equidad, sino también a la sociedad en general, pues esto se traduciría en mayor número de profesionales al servicio de la salud y significaría, además, igualdad en la construcción de una sociedad más incluyente.

\section{Referencias}

1. Mendes M, De Almeida D. La teoría de los roles en el contexto de la enfermería. Index Enferm. 2009; 18(4): 258-62. [citado 2020 febrero 15]. Disponible en: http:// scielo.isciii.es/scielo. php?script=sci_arttext\&pid=\$1132-12962009000400010

2. Cottingham M. The missing and needed male nurse: Discursive hybridization in professional nursing texts. Gender Work Organ. 2019; 26: 197-213. [citado 2020 enero 5]. Disponible en: https://doi.org/10.1111/gwao.12333

3. Torre M. Stopgappers? The Occupational Trajectories of Men in Female-Dominated Occupations. Work and Occupations. 2018; 45(3): 283-312. [citado 2020 abril 9]. Disponible en: https://doi.org/10.1177/0730888418780433

4. Whitford H, Marland G, Carson M, Bain H, Eccles J, Lee J, et al. An exploration of the influences on under representation of male preregistration nursing students. Nurse Education Today. 2020; 4: 1-5. [citado 2020 mayo 12]. Disponible en: https://doi.org/10.1016/i. nedt.2019.104234

5. Dal Sasso, Karina, De Campos Renata, Galvão, Cristina. Revisão integrativa: método de pesquisa para a incorporação de evidências na saúde e na enfermagem. Texto \& Contexto - Enfermagem. 2008;17(4):758-64.

6. Collière M. Promover la vida. 2th ed. México: McGraw-Hill Interamericana; 2009. Origen de las prácticas de cuidadoras; su influencia en la práctica de la Enfermería; p. 5-13.

7. Martínez M, Chamorro E. Historia de la enfermería Evolución del cuidado enfermero. 3rd ed. España: Elsevier; 2017. Etapa vocacional del cuidado; p. 67-125.

8. Sánchez M. El Hospital de San Lázaro de la ciudad de México y los leprosos novohispanos durante la segunda mitad del siglo XVIII. 0. 2010;42:81113. [citado 2020 diciembre 12]. Disponible en: http://dx.doi.org/10.22201/ iih.24486922e.2010.042.18441

9. González D. Nicholas MORTON: The Teutonic Knights in the Holy Land, 11901291. RUHM. 2017; 7(15): 242. Disponible en: https://ruhm.es/index.php/RUHM/ article/view/479.

10. Muench $U$, Dietrich $H$. The male-female earnings gap for nurses in Germany: A pooled cross-sectional study of the years 2006 and 2012. International Journal of Nursing Studies [Internet]. 2017 [citado 2020 diciembre 21]; 89: 125-131. Disponible en: http://www.sciencedirect.com/science/article/pii/ S0020748917301566 
11. Negro L. San Camilo de Lelis. Soldado de las galeras del Papa, de Venecia y de España. Revista de Historia Naval. 2018; 36(143): 69-92. [citado 2020 diciembre 22]. Disponible en: https:// dialnet.unirioja.es/servlet/articulo?codigo $=7533985$

12. O'Lynn C. Men in Nursing History, Challenges, and Opportunities. Estados Unidos: Springer Publishing Company; 2007. The Dark Ages of Nursing: The Disappearance of Men Nursing?; p. 22-3.

13. Rojas J, Siles J. Perspectiva disciplinar de enfermería en la obra de San Pedro Claver. Cult cuid. 2010; 14(27): 54-60. [citado 2020 noviembre 2]. Disponible en: https://doi.org/10.7184/ cuid.2010.27.07

14. Velandia A. Influencia de la "Cuestión Femenina" en la educación de enfermería en Colombia. Investigación y Educación en Enfermería. 2002; 20(1): 36-52. [citado 2020 noviembre 3]. Disponible en: https://revistas.udea.edu.co/index.php/ iee/article/view/16741

15. Sullivan L. The Education of Black Health Professionals. Phylon. 1977; 38 (2):18193. [citado 2020 septiembre 4]. Disponible en: https://doi.org/10.2307/274681

16. Naranjo Y. Reflexiones conceptuales sobre teorías de Enfermería y su validez en la práctica cubana. Rev. Cubana Enf. 2016; 32(4): 126-38. [citado 2020 septiembre 15]. Disponible en: http://www.revenfermeria.sld.cu/index.php/enf/article/view/986

17. Pérez R, Debasa F. La acción social y humanitaria de las Fuerzas Armadas. Madrid: Dykinson; 2019. Henry Dunant y el ideal humanitario. La fundación de la cruz roja y los orígenes del derecho internacional humanitario; p. 25-46.

18. Siles J. Walt Whitman, Poesía y Cuidados. Cultura de los cuidados. 2015; 43:12-8. [citado 2021 enero 14]. Disponible en: http://dx.doi.org/10.14198/ cuid.2015.43.02

19. The American Association for Men in Nursing AAMN. Advancing Men in Nursing [Internet]. 2021. [citado 2020 agosto 4]. Disponible en: https:// www.aamn.org/.

20. Ashkenazi L, Livshiz-Riven I, Romem P, Grinstein-Cohen O. Male Nurses in Israel: Barriers, Motivation, and How They Are Perceived by Nursing Students. J Prof Nurs Off J Am Assoc Coll Nurs. Abril de 2017; 33(2): 162-9.

21. Turcotte P, Bergheul S, Dubé N. Hombres estudiando en profesiones conceptuadas como femeninas en Quebec. Revista Internacional de Ciencias Sociales. 2017; 6(1): 1-10. [citado 2020 agosto 5]. Disponible en: https:// journals.eagora.org/revSOCIAL/article/view/56/944

22. Cottingham M. Caring moments and their men: masculine emotion practice in nursing. NORMA. 2017; 12(3-4): 270-85. [citado 2020 septiembre 15]. Disponible en: https://doi. org/10.1080/18902138.2017.1312954. 
23. Mott J. Professional male caregiving: A concept analysis. Nursing Forum. 2018; 53(4): 574-8. [citado 2020 enero 5]. Disponible en: https://doi. org/10.1111/nuf.12288

24. Palmer Troy. Barriers to Male Faculty in Nursing Education [Doctoral Dissertation]. [Minnesota]: Walden University; 2019. [citado 2020 septiembre 1]. Disponible en: https://scholarworks.waldenu.edu/dissertations/6470

25. Ntombizodwa N, Salaminah M. Four-year diploma male students' experiences in a profession traditionally perceived as a female domain at a selected public college of nursing in Limpopo, South Africa. Curationis. 2018; 41(1): e1-6. [citado 2020 septiembre 11]. Disponible en: https://www.ncbi. nlm.nih.gov/pmc/articles/PMC6191660/

26. Kristin J, Kristin H. Masculinity and nursing care: A narrative analysis of male students' stories about care. Nurse Education in Practice. 2015; 15(6): 409-14.

27. Carte N, Williams C. Role strain among male RNs in the critical care setting: Perceptions of an unfriendly workplace. Intensive Crit Care Nurs. 2017; 43: 81-6. [citado 2020 febrero 2]. Disponible en: https://pubmed. ncbi.nlm.nih.gov/28969956/

28. Valenzuela A, Keijzer B. Masculinidades en profesiones femeninas de Salud y Ciencias Sociales. Chile: Universidad Central; 2015. Concepto de masculinidad. p. 33-5.

29. Castrillón M. Formación universitaria de enfermeras Colombia $1937-$ 1980. Investigación y Educación en Enfermería. 1986;53-72. [citado 2020 abril 13]. Disponible en: https://revistas.udea.edu.co/index.php/iee/article/view/22640

30. Colombia. Congreso de la República. Ley 266, por la cual se reglamenta la profesión de enfermería en Colombia y se dictan otras disposiciones. Diario Oficial, 42.710 de febrero de 1996. https://www.mineducacion.gov. co/1759/articles-105002 archivo pdf.pdf

31. Colombia. Congreso de la República. Ley 911, Por la cual se dictan disposiciones en materia de responsabilidad deontológica para el ejercicio de la profesión de Enfermería en Colombia; se establece el régimen disciplinario correspondiente y se dictan otras disposiciones. Diario Oficial, 45.693 de octubre de 2004. https://www.mineducacion.gov.co/1621/articles-105034 archivo pdf.pdf

32. Consejo Técnico Nacional de Enfermería. Política Nacional de Talento Humano en Enfermería y Plan Estratégico 2020-2030. Colombia: Ministerio de Salud y Protección Social; 2020. Enfoques de humanización del trabajo en enfermería; p. 27-8. 
33. Brookes N. Modelos y teorías en enfermería. 3rd ed. Barcelona: Elsevier; 2015. El modelo de la marea en la recuperación de la salud mental; $p$. 610-39.

34. Scotto C. Modelos y teorías. 3rd ed. Barcelona: Elsevier; 2015. Teoría bioética sinfonológica; p. 505-19.

35. O'Lynn C, Tranbarger R. Men in Nursing. History, Challenges, and Opportunities. New York: Springer Publishing Company; 2007. 3 p.

36. Tranbarger R. Tranbarger R. Men in Nursing History, Challenges, and Opportunities. $1^{\circ}$ Edition. New York: Springer Publishing Company; 2007. The American Assembly for Men in Nursing (AAMN): The First 30 Years as Reported in Interaction; p. 67-82.

37. Zhang W, Liu Y. Demonstration of caring by males in clinical practice: A literature review. Int J Nurs Sci. 1 de septiembre de 2016; 3(3): 323-7. [citado 2020 mayo 24]. Disponible en: http://www.sciencedirect.com/science/ article/pii/ S2352013215300351

38. Lázaro S, Cabello B. Influencia de los estereotipos de género en los hombres profesionales de enfermería [Trabajo de grado]. [Madrid, España]: Universidad Autónoma de Madrid; 2018 [citado 2020 junio 26]. Disponible en: https://repositorio.uam.es/handle/10486/685191

39. McDowell J. Talk in feminized occupations: exploring male nurses' linguistic behaviour. Gender and Language. 2015;9(3):365-89. [citado 2020 septiembre 28]. Disponible en: https://pubmed.ncbi.nlm.nih.gov/28969956/

40. Carte N, Williams C. Role strain among male RNs in the critical care setting: Perceptions of an unfriendly workplace. Intensive Crit Care Nurs. 2017; 43:81-6. [citado 2020 octubre 03]. Disponible en: https://pubmed. ncbi.nlm. nih.gov/28969956/

41. Aspiazu E. Las condiciones laborales de las y los enfermeros en Argentina: entre la profesionalización y la precariedad del cuidado en la salud. Trabajo y sociedad. 2017; 28: 11-35. [citado 2020 enero 12]. Disponible en: http://www.scielo. org.ar/scielo.php?script=sci arttext\&pid=S1514$\underline{68712017000100002 \& \operatorname{lng}=e s \& n r m=i s o \& t \operatorname{lng}=e s}$

42. Osorio J. Par evaluador reconocido por Minciencias [Internet]. CVLAC. 2021. [citado 2021 abril 13]. Disponible en: https://scienti.minciencias. gov.co/cvlac/visualizador/generarCurriculoCv.do?cod rh=0001412161

43. Duque C. Hoja de vida [Internet]. CvLAC. 2021 [citado 2021 abril 14]. Disponible en: https://scienti.minciencias.gov.co/cvlac/visualizador/generarCurriculoCv.do?cod $r h=0000065956$ 
44. Quintero V. Hoja de Vida [Internet]. CvLAC. 2021 [citado 2021 abril 14]. Disponible en: http://scienti.colciencias.gov.co:8081/cvlac/visualizador/generarCurriculoCv.do?cod rh=0001557265

45. Rojas J. Hoja de Vida [Internet]. 2020. [citado 2021 abril 13]. Disponible en: http://www. udea.edu.co/wps/wcm/connect/udea/951f5c6a-d655-4204-8d27-7d8ea3f676b0/Hoja+de+vida+Juan+Rojas. pdf?MOD=AJPERES\&CVID=mBUJAHY

46. Velosa L. Hoja de Vida [Internet]. Hoja de Vida. 2021 [citado 2021 abril 14]. Disponible en: https://scienti.minciencias.gov.co/cvlac/visualizador/generarCurriculoCv.do?cod rh=0000069304 